TRANSLATING NEUROSCIENCE TO THE FRONT LINES: POINT-OF-CARE DETECTION OF NEUROPSYCHIATRIC DISORDERS

Jayashree Dasgupta*, Supriya Bhavnani,*, Georgia Lockwood Estrin, Debarati Mukherjee, Arpan Banerjee, Matthew K Belmonte, Bhismadev Chakrabarti, Gauri Divan, Geraldine Dawson, Mark H Johnson, James C McPartland, Nandini C Singh, Vikram Patel**

Centre for Chronic Conditions and Injuries, Public Health Foundation of India, Gurgaon, India (J Dasgupta PhD, S Bhavnani PhD, D Mukherjee PhD, Prof V Patel FMedSci)

Centre for Global Mental Health, London School of Hygiene and Tropical Medicine, London, UK (G Lockwood Estrin PhD, Prof V Patel)

National Brain Research Centre, Manesar, India (Prof N C Singh PhD, A Banerjee PhD)

Department of Psychology, University of Reading, Reading, UK (B Chakrabarti PhD)

Nottingham Trent University, Nottingham, UK (M K Belmonte PhD)

Sangath, Goa, India (G Divan MRCPCH)

Centre for Brain and Cognitive Development, Birkbeck College, London UK (Prof M H Johnson $\mathrm{PhD}$ )

Departments of Psychiatry and Behavioral Sciences, Pediatrics, and Psychology and Neuroscience, Duke Center for Autism and Brain Development, Duke University, North Carolina, USA (Prof G Dawson PhD)

Yale Child Study Center, Yale School of Medicine, New Haven, CT, USA (J C McPartland $\mathrm{PhD})$

* Contributed equally

**Corresponding author

Correspondence to:

Prof Vikram Patel

Public Health Foundation of India

Plot no 47, Sector 44

Gurgaon - 122002

vikram.patel@1shtm.ac.uk

+919822132038

Word count: 991 words 
A key challenge facing global mental health has been addressing the large 'treatment gaps' for neuropsychiatric disorders, to which large 'detection gaps' contribute significantly. The majority of these conditions are diagnosed either very late, or not at all. ${ }^{1}$ Low- and middle-income countries (LMICs) in particular present three barriers: low help-seeking behavior, in part due to limited awareness and stigma associated with mental disorders; paucity of mental health professionals to conduct diagnostic assessments; and reliance on diagnostic interviews many of which are time-intensive or proprietary and may not be cross-culturally valid. ${ }^{2}$ Identifying biomarkers that could aid early detection of mental disorders is a major goal for neuroscience research. Biomarkers may be defined as "any characteristic that is objectively measured and evaluated as an indicator of normal biological processes, pathogenic processes, or pharmacologic responses to a therapeutic intervention". ${ }^{3}$ Identification and characterization of biomarkers offers a tantalizing new translational opportunity to address detection gaps in LMICs by enabling pointof-care detection by non-specialist health workers. We illustrate our proposal through the example of Autism Spectrum Disorders (ASD).

The priority placed on early detection of ASD has been recognized by high-income countries' (HICs) development of large consortia that integrate multidisciplinary expertise for biomarker discovery, such as the EU-AIMS (www.eu-aims.eu) and the ABC-CT (www.asdbiomarkers.org). A variety of biomarkers, including genetic variations, proteomic and metabolic profiles, brain anatomy and function, and markers of cognitive and behavioral deficits offer promise in discriminating typically developing children from those with ASD. ${ }^{4}$ For LMICs to benefit from such research, however, biomarker screening and detection methods must be low-cost, scalable, acceptable to the end user and feasible for use in community settings and for delivery by nonspecialist frontline workers. Additionally, selected biomarkers must be highly sensitive and specific, being able to identify children with ASD during community-level screening, while limiting the number of false positives to avoid straining the limited health resources in LMICs and minimizing adverse social consequences to those who do not have this condition. Such 'point-of-care' detection tools would pave the way for targeted referral to specialists for confirmatory diagnostic assessments and early intervention programs.

Among direct child assessment measures, atypical eye gaze is one of the most promising biomarkers of ASD. ${ }^{5}$ Various measures of neural connectivity and specific neural functions are also associated with ASD. ${ }^{6}$ Bearing scalability in mind, portable eye-tracking technology and electroencephalography currently have the most promise for ASD screening. These technologies are non-invasive, applicable from infancy to adulthood and can be acquired on commercially available, portable platforms. While near infrared spectroscopy shares some of these advantages, there is currently limited evidence available to inform on its potential for use in detection of ASD. LMICs can assess the potential of these promising biomarkers when evaluated on rapidly evolving portable hardware, in less controlled recording environments, administered by nonspecialist community workers.

Although complex interactions between genetic vulnerability and environmental factors have long been viewed as being causal to ASD, apart from a few single-gene disorders, no common set of loci can reliably predict ASD. ${ }^{4}$ The identification of biomarkers in peripheral blood, such as altered serotonin and glutamate and GABA levels, ${ }^{7}$ could be particularly useful because the logistics of cold-chain transport of biomaterials to analytical laboratories have been established in most countries. Currently, the requirement of state-of-the-art, expensive, laboratory-based genomic and proteomic technologies to assess these biomarkers poses a limitation in LMICs; 
however, as with polymerase chain reaction technologies for the diagnosis of infectious diseases, there is a potential for investment in their adaptation for scalability if diagnostic properties prove to be robust.

Mobile health (mHealth) platforms have immense potential to address the multiple challenges illustrated above. ${ }^{8}$ In the context of ASD, while there are a number of tablet-based interventions readily available, ${ }^{9}$ there are relatively few tablet-based detection tools. For instance, screening questionnaires such as the Modified Checklist for Autism in Toddlers and the Social Communication Disorder Checklist, which are now being used routinely in some HICs, can readily be adapted onto mobile platforms from traditional paper-pencil methods, containing programmed algorithms for scoring. A first step to such mHealth adaptations is to ensure availability of culturally validated tools for screening and diagnosis in local languages. These could be supplemented by videos demonstrating typical and ASD behavioural biomarkers to assist parents in diverse cultural contexts to respond accurately to questions (http://asdetect.org/) . Mobile applications containing automatic coding and analysis of video recordings of a child's facial expressions are also being currently tested. ${ }^{10}$ Eye-tracking assessments could also be fully integrated within such mHealth platforms.

While ASD cannot yet be definitively differentiated from typical development in an individual on the basis of any single biomarker, mHealth platforms offer the potential of combining and triangulating information from assessment of multiple biomarkers. Bayesian statistical models can be used to estimate the probability of 'caseness' based on findings drawn from a combination of assessment methods to improve the accuracy of ASD risk prediction (Figure 1). In addition, the integration of big data analytics and machine learning algorithms during development of such a screening platform will allow refinement of screening algorithms through feedback of data on the actual diagnosis of the individual. Such data from large-scale population-based surveillance using an integrated mHealth platform may ultimately provide the evidence base for development of a point-of-care diagnostic tool for ASD.

Despite a growing body of evidence on ASD biomarkers, and similar research in other mental disorders, data on their application at the community level or in LMICs are limited. In parallel, technologies need to evolve to fulfil the criteria of scalability and demonstrate potential for use in natural, uncontrolled settings. Such advances also would benefit low-resource settings within HICs. Further, the expansion of research initiatives to LMIC populations would allow evaluation of biomarkers at global scales, incorporating diverse genetic and socio-cultural milieu. This is an opportune time for neuroscientists, clinicians, public health researchers and engineers to establish collaborative networks to design innovative solutions for detection of mental disorders and to usher in a new era for global mental health. ${ }^{11}$

Declaration of interests: The authors report no conflicting interests 
Figure $1 \mathrm{~A}$
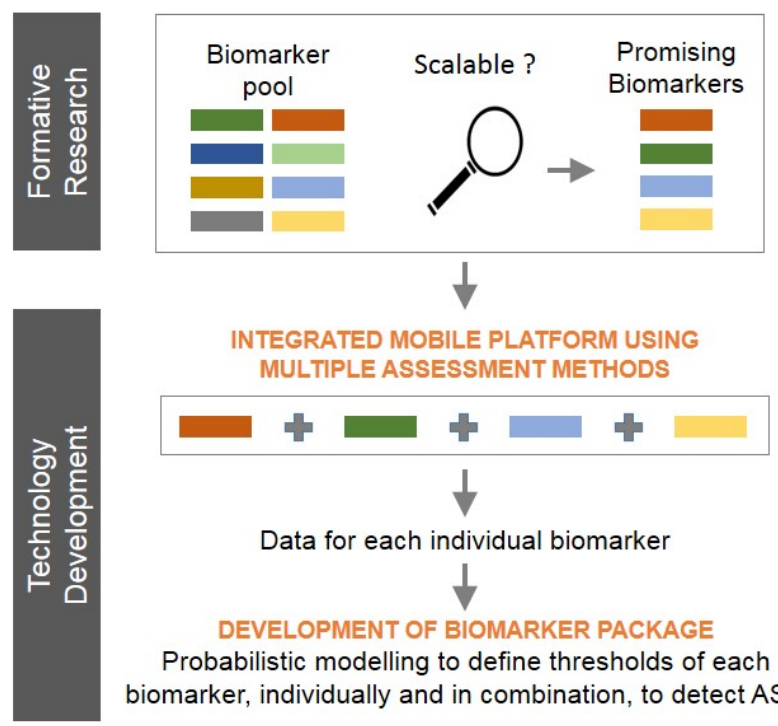

Data for each individual biomarker

$\downarrow$

DEVELOPMENT OF BIOMARKER PACKAGE

Probabilistic modelling to define thresholds of each biomarker, individually and in combination, to detect ASD

Figure 1B

Possible outcomes of 'Biomarker package'

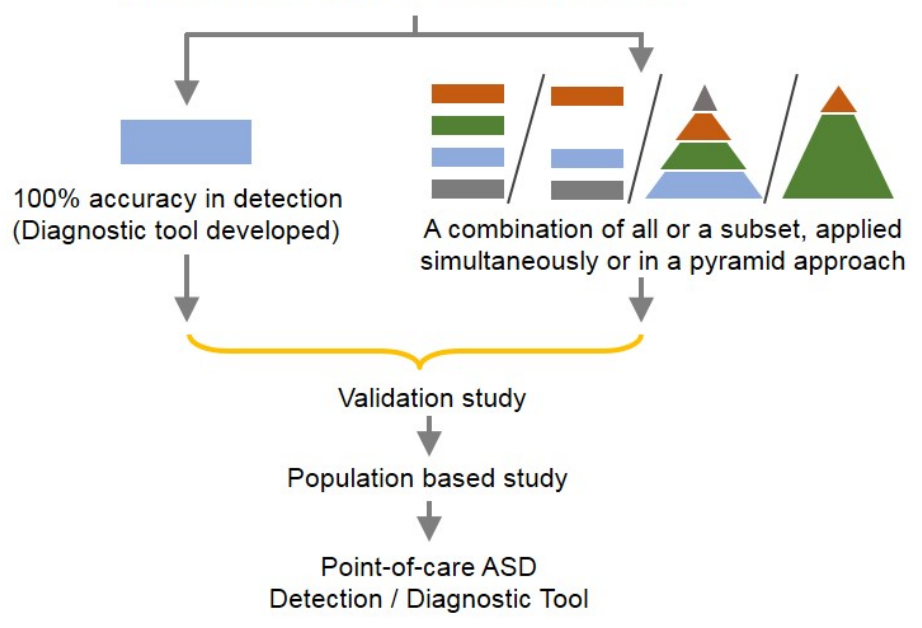




\section{Figure Legend}

\section{Figure 1: Development of point-of-care ASD detection/diagnostic tool}

a) Promising biomarkers, assessed using scalable technologies, can be integrated onto a mobile screening platform. Probabilistic modelling can identify thresholds for each biomarker individually and in combination to detect ASD.

b) Using scalable technologies, validation of the 'biomarker package' in large-scale population studies will allow machine learning algorithms to refine biomarker thresholds for a point-of-care diagnostic/detection tool for ASD.

\section{References:}

1 Whiteford HA, Ferrari AJ, Degenhardt L, Feigin V, Vos T. The global burden of mental, neurological and substance use disorders: an analysis from the Global Burden of Disease Study 2010. PLoS One 2015; 10: e0116820.

2 Durkin MS, Elsabbagh M, Barbaro J, et al. Autism screening and diagnosis in low resource settings: Challenges and opportunities to enhance research and services worldwide. Autism Res 2015; 8: 473-6.

3 Biomarkers and surrogate endpoints: Preferred definitions and conceptual framework. Clin. Pharmacol. Ther. 2001; 69: 89-95.

4 Walsh P, Elsabbagh M, Bolton P, Singh I. In search of biomarkers for autism: scientific, social and ethical challenges. Nat Rev Neurosci 2011; 12: 603-12.

5 Jones EJH, Gliga T, Bedford R, Charman T, Johnson MH. Developmental pathways to autism: a review of prospective studies of infants at risk. Neurosci Biobehav Rev 2014; 39: $1-33$.

6 Ecker C, Bookheimer SY, Murphy DGM. Neuroimaging in autism spectrum disorder: brain structure and function across the lifespan. Lancet Neurol 2015; 14: 1121-34.

7 Goldani AAS, Downs SR, Widjaja F, Lawton B, Hendren RL. Biomarkers in autism. Front psychiatry 2014; 5: 100.

8 Hurt K, Walker RJ, Campbell JA, Egede LE. mHealth Interventions in Low and MiddleIncome Countries: A Systematic Review. Glob J Health Sci 2016; 8: 54429.

9 Grynszpan O, Weiss PLT, Perez-Diaz F, Gal E. Innovative technology-based interventions for autism spectrum disorders: a meta-analysis. Autism 2014; 18: 346-61.

10 Campbell K, Hashemi J, Espinosa S, Marsan S, Schaich Borg J, Harris A, Chang Z, Qiu Q, Tepper M, Calderbank R, Baker JP, Sapiro G, Egger H DG. Computer Vision Detects Delayed Social Orienting in Toddlers with Autism. In: Presented in the International Society for Autism Research. Baltimore, MD, 2016.

11 Stein DJ, He Y, Phillips A, Sahakian BJ, Williams J, Patel V. Global mental health and neuroscience: potential synergies. The Lancet Psychiatry 2015; 2: 178-85. 